\title{
5-Hydroxymethylcytosine profiles of cfDNA are highly predictive of R-CHOP treatment response in diffuse large B cell lymphoma patients
}

Hang-Yu Chen ${ }^{1 \dagger}$, Wei-Long Zhang ${ }^{2 \dagger}$, Lei Zhang ${ }^{6}$, Ping Yang ${ }^{2}$, Fang Li ${ }^{2}$, Ze-Ruo Yang ${ }^{6}$, Jing Wang ${ }^{2}$, Meng Pang ${ }^{2}$,

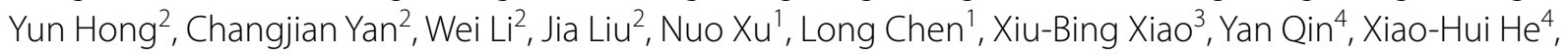

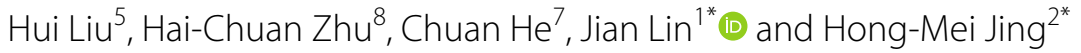

\begin{abstract}
Background: Although R-CHOP (rituximab, cyclophosphamide, doxorubicin, vincristine, and prednisone) remains the standard chemotherapy regimen for diffuse large B cell lymphoma (DLBCL) patients, not all patients are responsive to the scheme, and there is no effective method to predict treatment response.

Methods: We utilized $5 \mathrm{hmC}$-Seal to generate genome-wide $5 \mathrm{hmC}$ profiles in plasma cell-free DNA (cfDNA) from 86 DLBCL patients before they received R-CHOP chemotherapy. To investigate the correlation between $5 \mathrm{hmC}$ modifications and curative effectiveness, we separated patients into training $(n=56)$ and validation $(n=30)$ cohorts and developed a $5 \mathrm{hmC}$-based logistic regression model from the training cohort to predict the treatment response in the validation cohort.
\end{abstract}

Results: In this study, we identified thirteen $5 \mathrm{hmC}$ markers associated with treatment response. The prediction performance of the logistic regression model, achieving 0.82 sensitivity and 0.75 specificity ( $A \cup C=0.78$ ), was superior to existing clinical indicators, such as LDH and stage.

Conclusions: Our findings suggest that the $5 \mathrm{hmC}$ modifications in cfDNA at the time before R-CHOP treatment are associated with treatment response and that $5 \mathrm{hmC}$-Seal may potentially serve as a clinical-applicable, minimally invasive approach to predict R-CHOP treatment response for DLBCL patients.

Keywords: Epigenetics, 5-Hydroxymethylcytosine (5hmC), Diffuse large B cell lymphoma, R-CHOP, Logistic regression modeling

*Correspondence: linjian@pku.edu.cn; hongmeijing@bjmu.edu.cn ${ }^{\dagger}$ Hang-Yu Chen and Wei-Long Zhang have contributed equally to this work

${ }^{1}$ Synthetic and Functional Biomolecules Center, Beijing National Laboratory for Molecular Sciences, Key Laboratory of Bioorganic Chemistry and Molecular Engineering of Ministry of Education, College of Chemistry and Molecular Engineering, Innovation Center for Genomics, Peking University, Beijing 100871, People's Republic of China

${ }^{2}$ Department of Hematology, Lymphoma Research Center, Peking University Third Hospital, Beijing 100191, People's Republic of China

Full list of author information is available at the end of the article

\section{Introduction}

Diffuse large B cell lymphoma (DLBCL) is the primary type of invasive lymphoid tissue tumor, accounting for about $30 \%$ of non-Hodgkin's lymphoma [1]. Although the majority of the DLBCL patients are elderly patients, this disease is found in all ages [2]. Since rituximab (R) joined cyclophosphamide, adriamycin, vincristine, and prednisone $(\mathrm{CHOP})$ chemotherapy regimen ten years ago, the 
overall survival rate of DLBCL patients has improved significantly [3].

However, $30-50 \%$ of patients are not sensitive to this standard treatment [4], and exiting methods fail to predict the treatment response before R-CHOP treatment accurately or efficiently $[5,6]$. Currently, positron emission tomography (PET)-CT is the gold standard to evaluate the efficacy of different treatment regimens for DLBCL. However, it is generally used after the treatment and thus cannot predict the treatment response [7]. The International Prognostic Index (IPI) is the primary prognostic risk assessment method for DLBCL, especially in high-risk patients, and is used for R-CHOP chemotherapies [8-10]. However, IPI cannot accurately predict the therapeutic effect of R-CHOP in DLBCL patients [11]. Furthermore, recent studies have demonstrated that the detection of the apoptosis inhibitor, survivin [12], activationinduced cytidine deaminase (AID) [13], plasma miRNA [14], exosome miRNA [15], and genes polymorphism [16, 17], as well as the presence of CD3 and FoxP3 in the immune microenvironment [18], were all potential indicators of treatment efficacy in DLBCL patients. However, these predictors showed contradictory results that have not been well solved. Therefore, an accurate and effective method to predict the response of R-CHOP regimen is highly necessary.

In recent years, cell-free DNA (cfDNA) in the circulating blood, which carries genetic and epigenetic information from cells of origin, has emerged as a promising noninvasive approach for the diagnosis and prognosis in cancer [19]. 5-Methylcytosines (5mCs) of DNA is an important epigenetic feature that plays an important role in gene expression and cancer development [20]. Kristensen et al. [21] found that the methylation of DAPK1 in cfDNA from patients with DLBCL can be used to assess the effect of R-CHOP treatment. In the human genome, 5 -methylcytosines $(5 \mathrm{mCs})$ in cfDNA are dynamic and reversible $[22,23]$ and can be oxidized into 5-hydroxymethylcytosines $(5 \mathrm{hmCs})$ through the ten-eleven translocation (TET) enzymes in an active DNA-demethylation process $[24,25]$. Therefore, $5 \mathrm{hmC}$, as an oxidation product of DNA demethylation $(5 \mathrm{mC})$, may also be used to assess the effect of R-CHOP treatment. Recently, a study has also shown that $5 \mathrm{hmC}$ is associated with the prognosis of DLBCL [26]. However, its role in the prediction of treatment response of R-CHOP scheme for DLBCL patients is not established.

In this study, we used $5 \mathrm{hmC}$-Seal technique to obtain genome-wide $5 \mathrm{hmC}$ profiles in plasma cfDNA from 86 DLBCL patients, before they received R-CHOP chemotherapy. Our results demonstrated that responders and non-responders of R-CHOP treatment had distinct $5 \mathrm{hmC}$ profiles and that $5 \mathrm{hmC}$ markers selected by bioinformatics tools and machine learning algorithms could be used to predict treatment response of $\mathrm{R}-\mathrm{CHOP}$ treatment in DLBCL patients.

\section{Materials and methods Study participants}

From 2017 to 2019, 86 diffuse large B cell lymphoma (DLBCL) patients from multicenter studies including Peking University Third Hospital, Fifth Medical Center of PLA General Hospital, and Cancer Hospital Chinese Academy of Medical Sciences were included in this study. All patients had signed the patient consent form. In all cases, the diagnosis of DLBCL was made using appropriate diagnostic criteria from the 2016 WHO classification of lymphoid tumors with combinations of histologic, immunohistochemical, and cell of origin (coo) defined according to the Hans algorithm [27]. Medical records were reviewed for demographic and clinical data. Laboratory tests, white blood cell count (WBC), renal and hepatic function examinations, lactate dehydrogenase $(\mathrm{LDH})$, and $\beta 2$ microglobulin ( $\beta 2 \mathrm{MG}$ ) and cfDNA from peripheral blood samples were collected before any treatment. Then, all patients received standard $\mathrm{R}-\mathrm{CHOP}$ chemotherapy. Other baseline assessments including bone marrow biopsy and PET/CT were conducted in all patients in the follow-up care. The disease stage was defined by the Ann Arbor staging system. Treatment efficacy was evaluated after four cycles of treatment according to Lugano 2014 criteria [28], and patients were divided into PD (progressive disease), SD (stable disease), PR (partial response), and CR (complete response) based on the treatment outcome. This study was conducted in accordance with the Declaration of Helsinki.

\section{Study design}

This study aimed to discover $5 \mathrm{hmC}$ markers to predict the curative effectiveness of R-CHOP scheme through high-efficiency hmC-Seal technology. Among the 86 patients recruited, PR and CR patients were grouped as responders $(n=57)$, and $\mathrm{PD}$ and SD patients were grouped as non-responders $(n=29)$ to $\mathrm{R}$-CHOP treatment. We split 86 patients into a training and validation cohort. The objective of the first part of the study was to screen candidate genes with differential $5 \mathrm{hmC}$ modifications in these two groups from the training cohort. The objective of the second part of the study was to predict treatment outcome, using the model developed in the first part, in the validation cohort (Fig. 1).

\section{Clinical samples collection and cfDNA preparation}

Eight milliliters of peripheral blood from DLBCL patients was collected into Cell-Free DNA Collection 


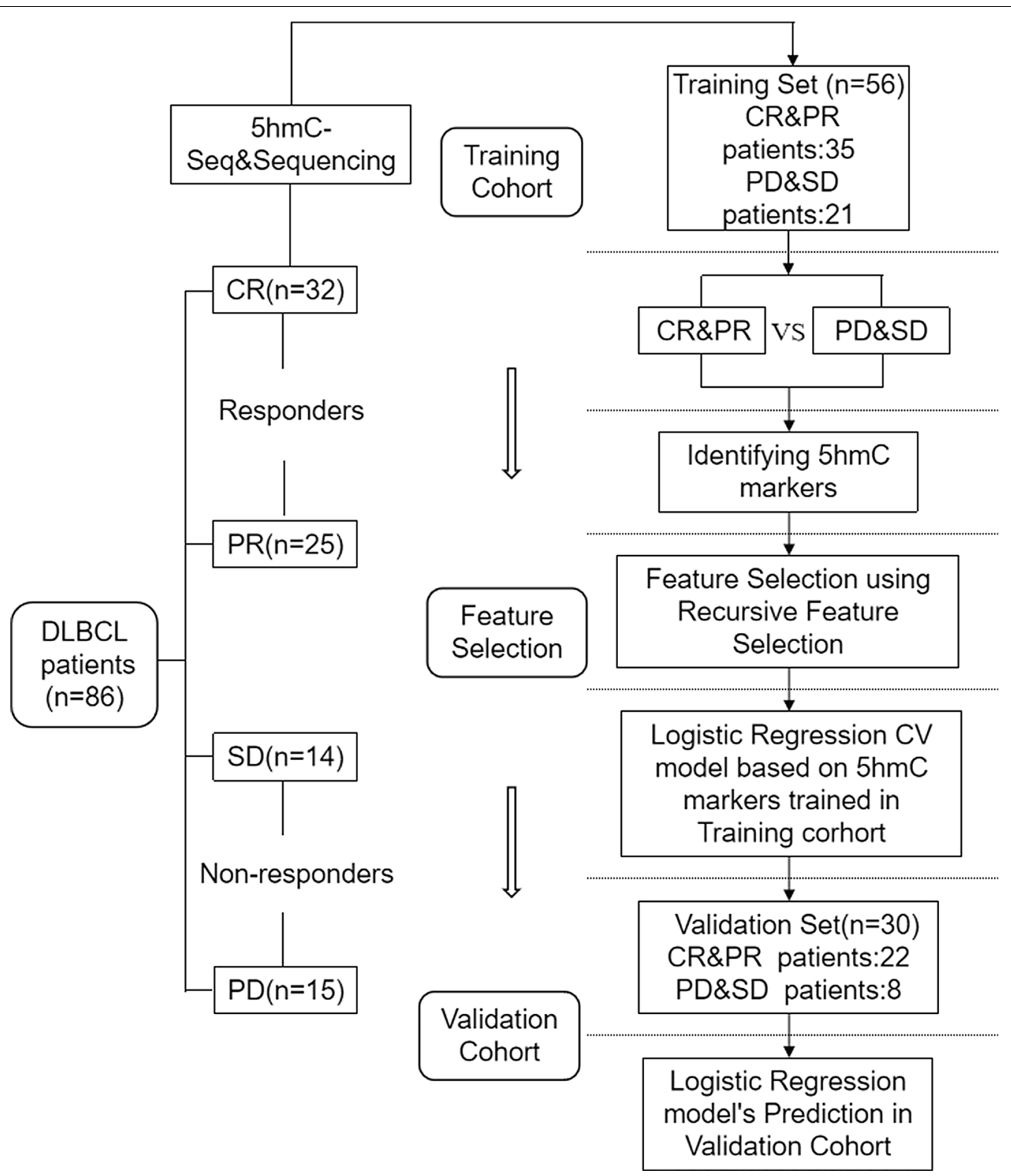

Fig. 1 Overview of study design. A total of 86 cfDNA samples were collected at the time of diagnosis from patients with DLBCL before R-CHOP or R-CHOP-like treatment. Based on treatment outcome in the follow-up care, patients were divided into the responder group (PR \& CR) and non-responder group (PD \& SD). A logistic regression model was trained by the training cohort that was used to predict treatment response in the validation cohort

Tubes (Roche). Within $24 \mathrm{~h}$, plasma was prepared by centrifuging twice at $1350 \times g$ for $12 \mathrm{~min}$ at $4{ }^{\circ} \mathrm{C}$ and $13,500 \times g$ for $12 \mathrm{~min}$ at $4{ }^{\circ} \mathrm{C}$. Then, the plasma samples were immediately stored at $-80^{\circ} \mathrm{C}$. The plasma cfDNA was extracted using the Quick-cfDNA Serum \& Plasma Kit (ZYMO) and then stored at $-80{ }^{\circ} \mathrm{C}$. The fragment size of all the cfDNA samples was verified by nucleic acid electrophoresis before library preparation.

\section{$5 \mathrm{hmC}$ library construction and high-throughput} sequencing

$5 \mathrm{hmC}$ libraries for all samples were constructed with high-efficiency hmC-Seal technology [29]. Due to the highly sensitive nature of the chemical labeling method, the input cfDNA can be as low as 1-10 ng. According to the requirements of next-generation sequencing, the cfDNA extracted from plasma was end-repaired, 3'-adenylated using the KAPA Hyper Prep Kit (KAPA 
Biosystems), and then ligated with the Illumina compatible adapters. The ligated cfDNA was added in a glycosylation reaction in $25 \mu \mathrm{L}$ solution containing $50 \mathrm{mM}$ HEPES buffer (pH 8.0), $25 \mathrm{mM} \mathrm{MgCl} 2,100 \mu \mathrm{M}$ UDP6-N3-Glc, and $1 \mu \mathrm{M} \beta$-glucosyltransferase (NEB) for $2 \mathrm{~h}$ at $37^{\circ} \mathrm{C}$. Next, the cfDNA was purified using DNA Clean \& Concentrator Kit (ZYMO). The purified DNA was incubated with $1 \mu \mathrm{L}$ of DBCO-PEG4-biotin (Click Chemistry Tools, $4.5 \mathrm{mM}$ stock in DMSO) for $2 \mathrm{~h}$ at $37^{\circ} \mathrm{C}$. Similarly, the DNA was purified using the DNA Clean \& Concentrator Kit (ZYMO). Meantime, $2.5 \mu \mathrm{L}$ streptavidin beads (Life Technologies) in $1 \times$ buffer (5 mM Tris pH 7.5, 0.5 mM EDTA, $1 \mathrm{M} \mathrm{NaCl}$, and $0.2 \%$ Tween 20) was added directly to the reaction for $30 \mathrm{~min}$ at room temperature. Finally, the beads were subsequently washed eight times for five minutes with buffer 1-4. All binding and washing steps were performed at room temperature with gentle rotation. Then, the beads were resuspended in RNase-free water and amplified with 14-16 cycles of PCR amplification. The PCR products were purified using AMPure XP beads (Beckman), according to the manufacturer's instructions. The concentration of libraries was measured with a Qubit 3.0 fluorometer (Life Technologies). Paired-end 39-bp high-throughput sequencing was performed on the NextSeq 500 platform.

\section{Mapping and identifying $5 \mathrm{hmC}$-enriched regions}

FastQC (version 0.11.5) was used to assess the sequence quality. Raw reads were aligned to the human genome (version hg19) with bowtie2 (version 2.2.9) [30] and further filtered with SAMtools (version 1.3.1) [31], (parameters used: SAMtools view -f 2 -F 1548 -q 30 and SAMtools rmdup) to retain unique non-duplicate matches to the genome. Pair-end reads were extended and converted into BedGraph format normalized to the total number of aligned reads using bedtools (version 2.19.1) [32], and then converted to bigwig format, using bedGraphToBigWig from the UCSC Genome Browser for visualization in the Integrated Genomics Viewer. Potential 5hmCenriched regions (hMRs) were identified using MACS (version 1.4.2), and the parameters used were macs 14 -p 1e-3 -f BAM -g hs [33]. Peak calls were merged using bedtools merge, and only those peak regions that appeared in more than 10 samples and that were less than $1000 \mathrm{bp}$ were retained. Blacklisted genomic regions that tend to show artifact signals, according to ENCODE, were also filtered. The hMRs for each patient were generated by intersecting the individual peak call file with the merged peak file. The hMRs within chromosome $\mathrm{X}$ and $\mathrm{Y}$ were excluded and used as an input for the downstream analyses.
Feature selection, model training, and validation

A two-step procedure was used to select optimal hMRs for distinguishing the non-responder group from the responder group prior to $\mathrm{R}-\mathrm{CHOP}$ treatment. In step 1 , DLBCL patients were randomly divided into training and validation cohorts in a stratified manner, using train_test_split in Scikit-Learn (version 0.22.1) [34] package in Python (version 3.6.10. In the training cohort, we identified differentially modified $5 \mathrm{hmC}$ regions (DhMRs) using EdgeR package (version 3.24.3) [35] in $\mathrm{R}$ (version 3.5.0), with the filtering threshold ( $p$ value $<0.01 \& \log$ 2FoldChange $>0.5$ ). In step 2, the dhMRs were further filtered using the recursive feature elimination algorithm (RFECV) in Scikit-Learn (parameters used: estimator $=$ LogisticRegressionCV (class_weight $=$ 'balanced', $\mathrm{cv}=2$, max_iter $=1000$ ), scoring ='accuracy').

Then, we trained the logistic regression CV model (LR) with the features selected from step 2 (parameter used: maxiter $=100$, method $=$ "lbfgs"). The trained LR model was used to predict the treatment outcome for patients in the validation cohort. Receiver operating characteristics (ROC) analysis was used to evaluate model performance. Area under the curve (AUC), best cutoff point, sensitivity, and specificity were computed with sklearn.metrics module.

\section{Exploring functional relevance of the $5 \mathrm{hmC}$ markers}

We annotated the dhMRs from step 1 using the ChIPseeker package (version 1.20.0) [36], and genes that were closest to the marker regions were used for the following functional analyses. The GO enrichment analysis (Biological Process) was done by the ClueGO (version 2.5.5) and CluePedia (version 1.5.5) plug-in from Cytoscape software (version 3.7.2) (parameters used: medium network specificity, Bonferroni step-down $\mathrm{pV}$ correction and two-sided hypergeometric test). We used the Search Tool for the Retrieval of Interacting Genes (STRING) database (version 10.0, https://string-db.org) to find protein-protein interactions for $5 \mathrm{hmC}$ markers. Then, the Cytoscape software was used to construct the PPI network.

\section{Survival analysis and gene expression correlation analysis in TCGA-DLBC}

For survival analysis, we downloaded the mRNA HTseqFPKM data of 48 DLBLC patients from the TCGA-DLBC dataset [37] in the GDC Data Portal using gdc-client (version 1.5.0) and downloaded manually curated clinical data, including overall survival (OS), disease-specific survival (DSS), disease-free interval (DFI), and progression-free interval (PFI) from UCSC Xena [38]. Survminer package (version 0.4.6) and Surviva packages (version 2.44-1.1) in $\mathrm{R}$ were used for survival analysis. Forty-eight 
patients were divided into the high-expression group and low-expression group according to the cutoff points determined by the maximally selected rank statistics algorithm (maxstat) [39]. Survival analysis of each gene was assessed by Kaplan-Meier curves [40] and the logrank test [41]. For the survival analysis, $p$ value $<0.05$ was considered statistically significant. For gene expression correlation analysis, we used a web tool called TIMER2.0 [42], which incorporated all TCGA expression data, to explore the mRNA expression relationship between $5 \mathrm{hmC}$ markers and other genes of interests in the TCGADLBC dataset. The correlation analysis was done using Spearman rank correlation.

\section{Statistical analysis}

For clinical data, continuous variables are presented as mean (SD) and categorical variables are presented as count (percentages). To understand the relationship between categorical/continuous variables and treatment outcome, Kruskal-Wallis test by ranks [43] and $\chi^{2}$ test [44] were used, respectively. A two-sided $p$ value of $<0.05$ was considered to indicate statistical significance. The predictive power of clinical data was estimated by glm function in R-base and pROC package (version 1.15.3) in R.

\section{Results}

\section{Clinical characteristics of Diffuse large B Cell lymphoma (DLBCL) patients}

The clinical summary, including baseline characteristics and laboratory data, of all 86 patients is shown in Table 1. Of the 86 patients with DLBCL patients, 46 were male and 40 were female. The median age of all the patients was 54.6 years, and $63.9 \%$ of patients had advanced disease (including stage III and stage IV). Importantly, all patients were newly diagnosed with DLBCL and received standard R-CHOP chemotherapy. Treatment efficacy was evaluated in all patients after 4 cycles of treatment. According to the efficacy standard of Lugano 2014 criteria, the treatment response of patients was as follows: CR in 32 patients (37.2\%), PR in 25 patients (29.1\%), SD in 14 patients (16.3\%), and PD in 15 patients (17.4\%). Besides, according to the Hans model, 23 patients (26.7\%) were germinal center B cell (GCB), 61 patients (70.9\%) had non-GCB and 2 patients (2.3\%) had an unknown cell of origin. The results of the international prognostic index (IPI) score showed that $52.3 \%$ of patients (IPI score $>2$ ) belonged to the high-intermediate-risk/high-risk group. Finally, the mean of WBC, LDH, and $\beta 2 \mathrm{MG}$ for all patients was $6.94 \times 10-4 / \mathrm{L}, 364.33 \mathrm{U} / \mathrm{L}$, and $2.84 \mathrm{mg} / \mathrm{L}$, respectively.
Table 1 Diffuse large B cell lymphoma (DLBCL) patient characteristics

\begin{tabular}{|c|c|c|}
\hline Characteristics & Level/type & Value \\
\hline$n$ & & 86 \\
\hline \multirow[t]{2}{*}{$\operatorname{Sex}(\%)$} & $\mathrm{F}$ & $40(46.5)$ \\
\hline & M & $46(53.5)$ \\
\hline Age (mean (SD)) & & $54.59(15.56)$ \\
\hline Diagnosis (\%) & DLBCL & $86(100.0)$ \\
\hline Therapy (\%) & $\mathrm{R}-\mathrm{CHOP}$ & $86(100.0)$ \\
\hline \multirow[t]{4}{*}{ Response (\%) } & $C R$ & $32(37.2)$ \\
\hline & PD & 15 (17.4) \\
\hline & $P R$ & $25(29.1)$ \\
\hline & SD & $14(16.3)$ \\
\hline \multirow[t]{5}{*}{ Ann Arbor stage (\%) } & 1 & $6(7.0)$ \\
\hline & $\|$ & $18(20.9)$ \\
\hline & III & $7(8.1)$ \\
\hline & IV & $48(55.8)$ \\
\hline & Unknown & $7(8.1)$ \\
\hline \multirow[t]{3}{*}{ Cell of origin (\%) } & GCB & $23(26.7)$ \\
\hline & Non-GCB & $61(70.9)$ \\
\hline & Unknown & $2(2.3)$ \\
\hline \multirow[t]{7}{*}{$|P|(\%)$} & 0 & $8(9.3)$ \\
\hline & 1 & $12(14.0)$ \\
\hline & 2 & $18(20.9)$ \\
\hline & 3 & $28(32.6)$ \\
\hline & 4 & $15(17.4)$ \\
\hline & 5 & $2(2.3)$ \\
\hline & Unknown & $3(3.5)$ \\
\hline Mean LDH (SD) & & $364.33(326.72)$ \\
\hline Mean $\beta 2 M G$ (SD) & & $2.84(2.77)$ \\
\hline
\end{tabular}

IPI, International Prognostic Index; GCB, germinal center B cell; CR, complete response; $P R$, partial response; $S D$, stable disease; $P D$, progressive disease; $L D H$, lactate dehydrogenase; $\beta 2 M G$, beta 2 microglobulin; WBC, white blood cell

\section{$5 \mathrm{hmC}$ profiles differ between responders and non-responders to R-CHOP treatment in the training cohort}

Eighty-six DLBCL patients were randomly divided into the training cohort $(\mathrm{n}=56)$ and validation cohort $(n=30)$ (Fig. 1). We used hmC-Seal to generate genome-wide $5 \mathrm{hmC}$ profiles for patients in the training set, including 35 responders and 21 non-responders to R-CHOP treatment. The overall $5 \mathrm{hmC}$ enrichment (all hMRs) was most common in intronic, intergenic, and promoter regions for both responders and non-responders, even though no statistically significant difference was found between these two groups for any genomic feature types (Fig. 2a). Meanwhile, we conducted differential analysis (EdgeR; $p<0.01$, fold change $>0.5$ ) and observed 205 DhMRs, including upregulate $(n=124)$ and downregulate $(n=81)$ regions in responders compared to nonresponders (Fig. 2b). For instance, FBXL4 (Fig. 2c) was 


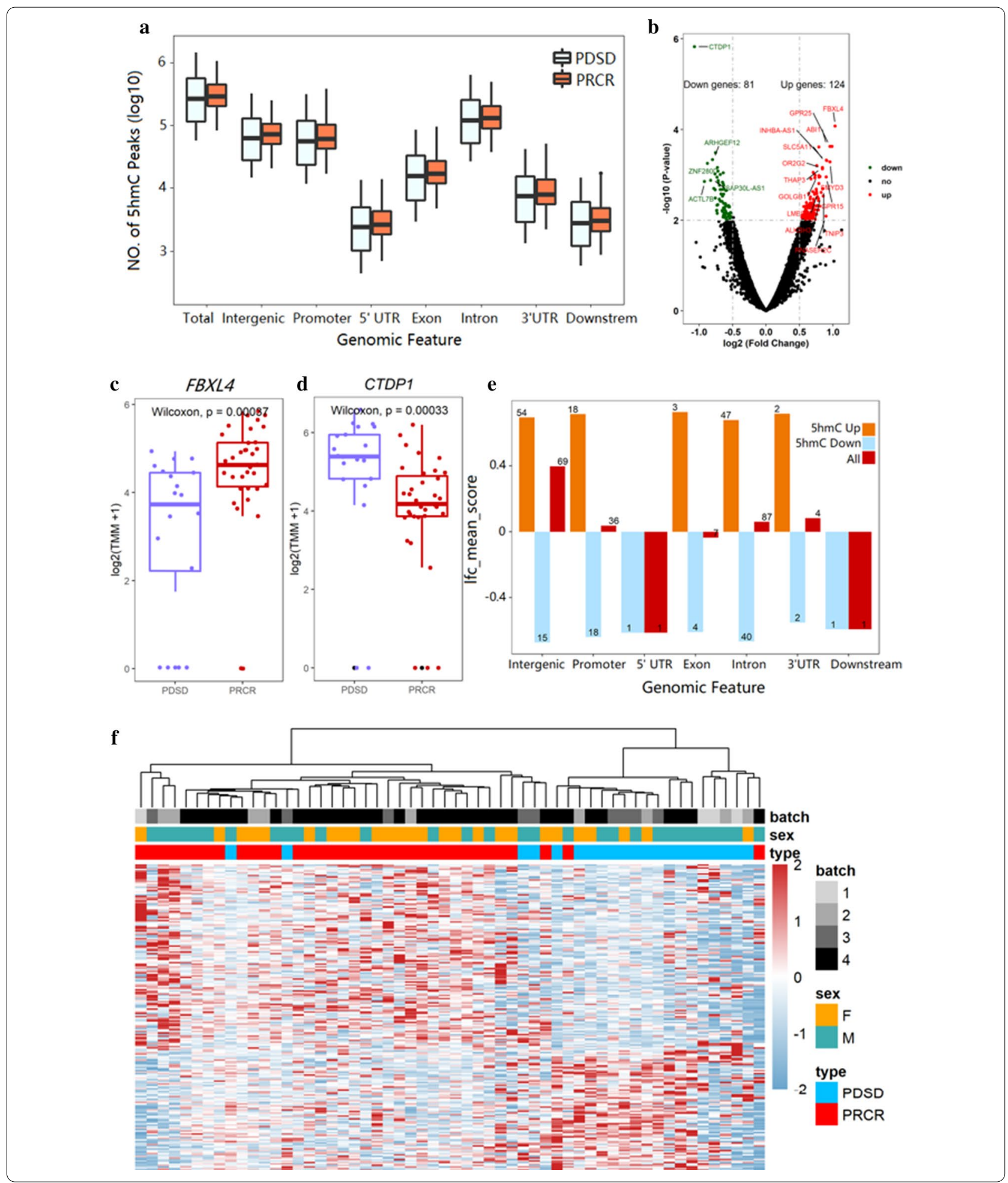

highly enriched in hydroxymethylation for responders $(p=0.00087)$, and CTDP1 (Fig. 2d) was highly enriched in hydroxymethylation for non-responders $(p=0.00033)$. In addition, for the top 205 DhMRs, the most significant enrichment was found in intronic, intergenic, and promoter regions, consistent with previous studies [45, 46] (Fig. 2e). Finally, heatmap results, using default clustering methods, demonstrated that these 205 DhMRs could effectively separate responders from non-responders (Fig. 2f). 
(See figure on previous page.)

Fig. 2 Characteristics of $5 \mathrm{hmC}$ distribution in plasma cfDNA of DLBCL patients in the training cohort $(n=56)$. a Genome-wide $5 \mathrm{hmC}$ distribution in different genomic features grouped by R-CHOP treatment response (PDSD vs PRCR). b Volcano plot. Significantly altered genes (abs (log2 Foldchange) $\geq 0.5 ; p$ value $<0.01$ ) are highlighted in red (up) or green (down) using the responder group (PRCR) as the reference $(n=205)$. Black dots represent the genes that are not differentially expressed. $\mathbf{c}$, $\mathbf{d}$ Boxplots of FBXL4 and CTDP1 grouped by treatment response (PDSD vs PRCR). Log2 transformed of TMM normalized $5 \mathrm{hmC}$ enrichment values were plotted, and the Wilcoxon $t$ test was used. e Mean log2 Foldchange value of 205 DhMRs across different genomic features (Orange for 124 5hmC-up DhMRs, blue for 81 5hmC-down DhMRs, red for all 205 DhMRs). f Heatmap of 205 DhMRs markers with treatment response, batch, and sex information labeled. Unsupervised hierarchical clustering was performed across genes and samples

\section{Pathway analysis and function exploration}

Pathway analysis of $2055 \mathrm{hmC}$ markers (Additional file 1: Table 1) in DLBCL patients suggested functional enrichment in certain canonical pathways. The top enriched
GO biological pathways included signaling like alphabeta $\mathrm{T}$ cell differentiation, protein-lysine $\mathrm{N}$-methyltransferase activity, and histone H3-K9 modification (Fig. 3a). Among these pathways, signaling by alpha-beta $\mathrm{T}$ cell

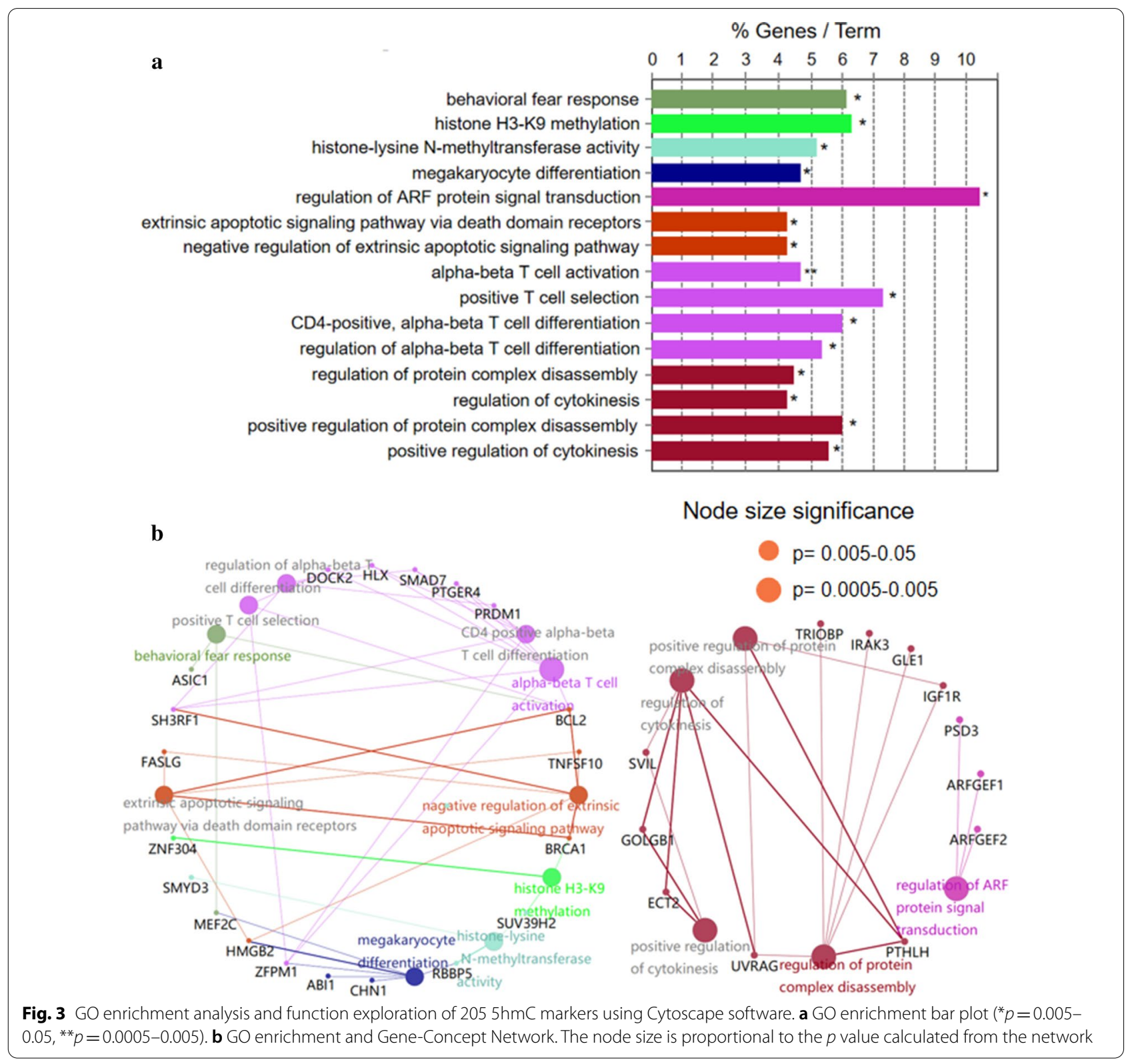


differentiation was known to be relevant to tumor growth and apoptosis, which suggested that the DhMRs might be involved in the immunity system [47-49]. Meanwhile, the hubs of the GO functional interaction networks (Fig. 3b) showed that these genes, including BCL2 apoptosis regulator (BCL2), PR/SET domain 1 (PRDM1), prostaglandin E receptor 4 (PTGER4), SMAD family member 7 (SMAD7), H2.0 like homeobox (HLX), dedicator of cytokinesis 2 (DOCK2) and SH3 domain containing ring finger 1 (SH3RF1), participated in the regulating $\mathrm{T}$ cell activation and differentiation pathway.

\section{5hmC markers showed prediction performance superior to clinical indicators for R-CHOP treatment response}

Similarly, we generated genome-wide $5 \mathrm{hmC}$ profiles for patients in the validation set, including 22 responders and 8 non-responders to R-CHOP treatment. By using the recursive feature elimination algorithm based on the logistic regression CV estimator, we further reduced the number of $5 \mathrm{hmC}$ markers from 205 to 13, which achieved the best cross-validation score (Additional file 2: Figure S1). Further, we found that the $135 \mathrm{hmC}$ markers (Table 2), selected by the LR model, could distinguish responders from non-responders in both the training and validation cohorts (Fig. 4a, b). Meantime, these $135 \mathrm{hmC}$ markers could effectively predict responders and non-responders to R-CHOP treatment in the training $(\mathrm{AUC}=1.00)$ and the validation cohorts $(\mathrm{AUC}=0.78)$ (Fig. 4c), achieving 0.82 sensitivity and 0.75 specificity in the validation cohort (Fig. 4d). Finally, we also calculated the individual AUC for each of the $135 \mathrm{hmC}$ markers in the training and validation cohorts (Additional file 2: Figure S2A, B). Among these, ARHGEF12 and ZNF280D showed the best predictive performance, yielding an AUC of 0.76 in the validation cohort.

We also investigated the association between available clinical indicators, including stage, pathology, IPI, LDH, $\beta 2 \mathrm{MG}$ and $\mathrm{WBC}$, and $\mathrm{R}-\mathrm{CHOP}$ treatment response. Among all those clinical indicators, only LDH (continuous variable, $p=0.03474$ ) and stage (categorical variable, $p=0.004453$ ) showed a significant association with treatment response (Additional file 2: Table 3). Thus, we used these two indicators to build logistic regression models to predict treatment response. As expected, LDH level, stage, and LDH combined with stage (LDH + stage) could also predict treatment response to a certain level. However, the AUC of LDH level (AUC=0.646), stage $(A U C=0.658)$, and $\mathrm{LDH}$ combined with stage $(\mathrm{AUC}=0.669)$ were lower than that of $5 \mathrm{hmC}$ markers $(\mathrm{AUC}=0.78)($ Fig. 4e).

\section{Potential associations between $5 \mathrm{hmC}$ markers and R-CHOP treatment response in DLBCL patients}

To further understand the potential associations between those 13 5hmC-modified marker genes and R-CHOP treatment response, we investigated their mRNA expression profiles and compared them to that of B-lymphocyte antigen CD20 (MS4A1), a rituximab target gene, in 48 DLBCL patients from the TCGA-DLBC dataset. Among those 13 marker genes, we found that the mRNA expression of MS4A1 was positively correlated with the mRNA expression of ARHGEF12 (rho=0.385), FBXL4 (rho=0.376), GOLGB1 (rho=0.434), LMBR1 $($ rho $=0.45)$ (Additional file 2 Figure S3A-D). We decided to further investigate the potential mechanism of Rho Guanine Nucleotide Exchange Factor 12 (ARHGEF12),

Table 2 Coefficients for $13 \mathbf{5 h m C}$ markers in the logistic regression model trained by the training cohort

\begin{tabular}{|c|c|c|c|c|c|}
\hline Markers & GenelD & Coefficients & SE & $z$ value & $p$ value \\
\hline Intercept & & -5.5704 & 0.867 & 2.652 & $<0.01$ \\
\hline chr1_6721489_6721898 & THAP3 & 0.7712 & 0.145 & 1.865 & $<0.05$ \\
\hline chr1_246290825_246291238 & SMYD3 & 0.39 & 0.149 & 1.955 & $<0.05$ \\
\hline chr1_247755954_247756505 & $\mathrm{OR} 2 \mathrm{G} 2$ & 3.1779 & 0.108 & 1.344 & $<0.05$ \\
\hline chr11_43905400_43905804 & ALKBH3 & 3.3423 & 0.128 & 1.306 & $<0.05$ \\
\hline chr11_65511519_65512429 & RNASEH2C & 1.5211 & 0.072 & 2.061 & $<0.05$ \\
\hline chr11_120211662_120212234 & ARHGEF12 & -3.8797 & 0.115 & -3.225 & $<0.001$ \\
\hline chr15_56982146_56982638 & ZNF280D & -1.2266 & 0.177 & -3.250 & $<0.001$ \\
\hline chr16_24916341_24916920 & SLC5A11 & 0.6683 & 0.076 & 0.149 & $<0.05$ \\
\hline chr18_77500908_77501376 & CTDP1 & -2.573 & 0.103 & -3.182 & $<0.001$ \\
\hline chr3_98270705_98271079 & GPR15 & 0.1052 & 0.167 & 2.348 & $<0.05$ \\
\hline chr3_121430838_121431239 & GOLGB1 & 0.8526 & 0.178 & 2.982 & $<0.01$ \\
\hline chr6_99461404_99461922 & FBXL4 & 1.7188 & 0.101 & 2.165 & $<0.05$ \\
\hline chr7_156700537_156701031 & LMBR1 & 1.0942 & 0.078 & 0.579 & $<0.05$ \\
\hline
\end{tabular}




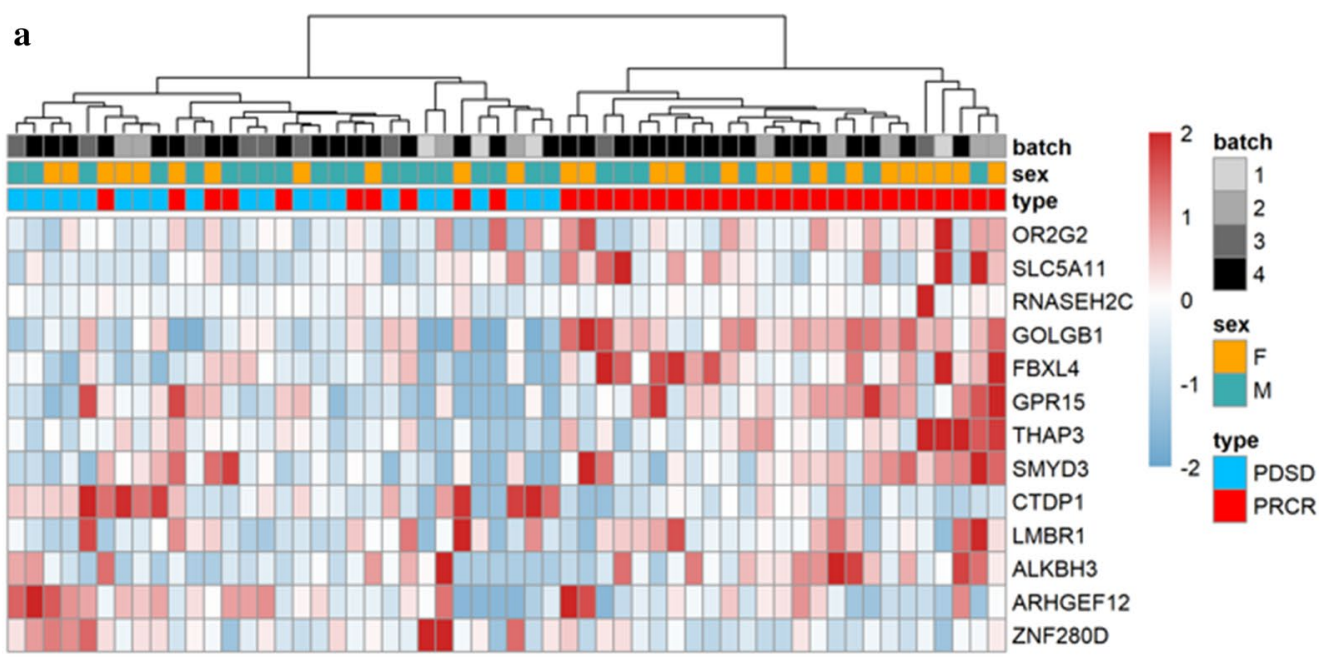

b

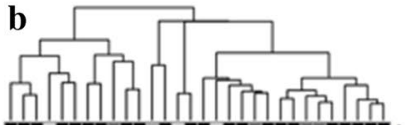

IIIn

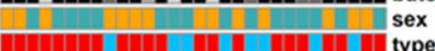
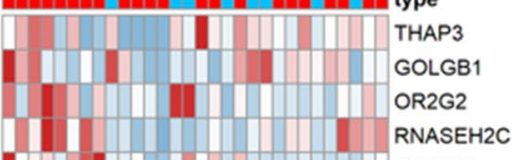

II 1 ALKBH3

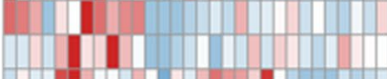

111.11 .2 LMBR1

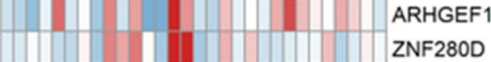

(1)

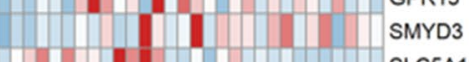

SLC5A11 c

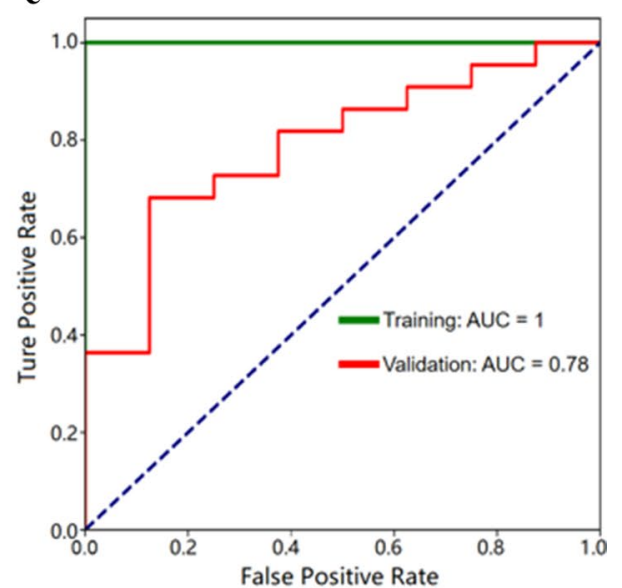

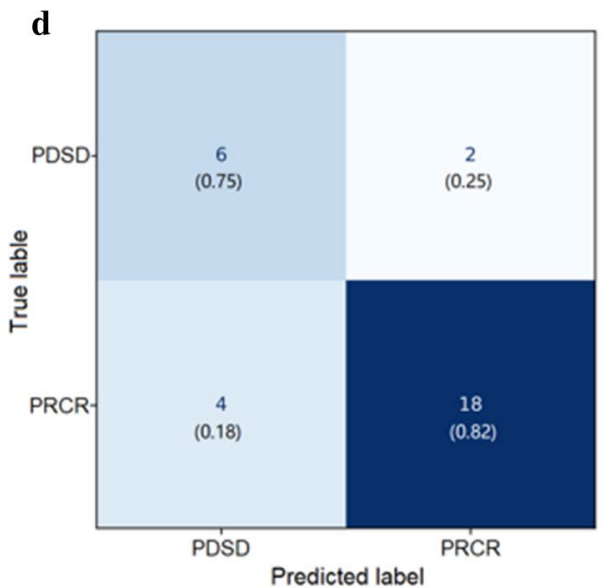
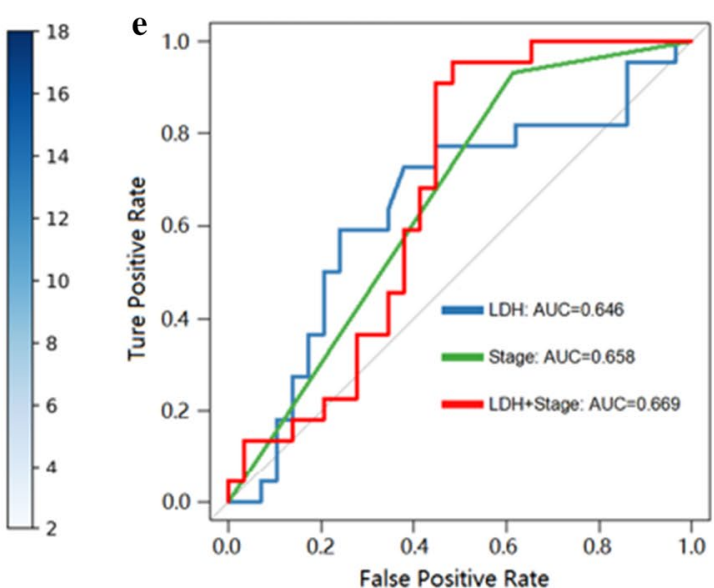

Fig. 4. $5 \mathrm{hmC}$ markers' prediction for treatment response in the training and validation cohort. a, b Heatmaps of $135 \mathrm{hmC}$ markers with treatment response, batch and sex information labeled in the training and validation cohorts. Unsupervised hierarchical clustering was performed across genes and samples. $\mathbf{c}$ Receiver operating characteristic (ROC) curve of the classification model with $135 \mathrm{hmC}$ markers in the training and validation cohorts. The true-positive rate (sensitivity) is plotted in function of the false-positive rate (1-specificity). $\mathbf{d}$ Confusion matrix that shows the model performance in the validation cohort (responders: 22 , non-responders:8). e ROC curve of the classification model with LDH, stage and LDH combined with stage for DLBCL patients 
for its mRNA expression was positively associated with $M S 4 A 1$, and it achieved the highest AUC in the validation cohort among $135 \mathrm{hmC}$-modified marker genes. According to recent studies, $5 \mathrm{hmC}$ enrichment in promoter regions was positively associated with gene expression levels $[25,50]$. In our study, ARHGEF12 was highly enriched in hydroxymethylation in the non-responders $(p=0.022)$ (Fig. 5a), and the hydroxymethylation site was in the promoter region (Additional file 3: Table 2). Therefore, we speculated that the change in $5 \mathrm{hmC}$ enrichment in the promoter region of ARHGEF12 might lead to the change in the mRNA expression of this gene.

In addition, from the PPI network constructed from the STRING database, we identified several genes linked to $A R H G E F 12$, including Ras Homolog Family Member A $(R H O A)$, Ras Homolog Family Member B (RHOB), Ras Homolog Family Member C (RHOC), Cell Division Cycle 42 (CDC42), Rho Associated Coiled-Coil Containing Protein Kinase 1 (ROCK1), G Protein Subunit Alpha 12 (GNA12) and G Protein Subunit Alpha 13 (GNA13) (Fig. 5b). Interestingly, we found that all of these gene expressions $(R H O A(\mathrm{rho}=0.667), R H O B(\mathrm{rho}=0.604)$, CDC42 (rho =0.676), ROCK1 $(\mathrm{rho}=0.832), \quad$ GNA12 $($ rho $=0.721)$, GNA13 $($ rho $=0.784))$ were highly positively associated with that of ARHGEF12 (Fig. 5c-h). Moreover, from survival analysis results in the TCGADLBC dataset, we found that the overall survival time (OS, days) of patients with high expression of ARHGEF12 and $C D C 42$ was significantly lower than that of patients with low expression in these 2 genes (Fig. 5i, j). Also, we found that the mRNA expression of ARHGEF12 was positively associated with several immune-related genes, such as CD44, CD47, CD53, CD59, and CD274 (Additional file 2: Figure S4A-E). Finally, we conducted a GO enrichment analysis (Fig. 5k) for all the genes associated with ARHGEF12 (Fig. 5b) and found that the main GO enrichment was in the Rho signaling pathway which was consistent with the PPI network constructed from the STRING database.

\section{Discussion}

Even though previous studies have reported that $5 \mathrm{hmC}$ modifications could serve as potential diagnostic and prognostic markers in DLBCL patients [26], its role in the prediction of treatment response of $\mathrm{R}-\mathrm{CHOP}$ scheme was not fully studied. Therefore, an accurate, noninvasive prediction test for treatment response of $\mathrm{R}-\mathrm{CHOP}$ is highly desirable, and to this end, the emergence of liquid biopsy technology has shown to be a promising approach. In this study, we aimed to develop a model to predict $\mathrm{R}$-CHOP scheme treatment response for DLBCL patients based on the $5 \mathrm{hmC}$ profiles derived from plasma cfDNA before $\mathrm{R}$-CHOP treatment using hmC-Seal sequencing method.

In our cohort, we found that responders and nonresponders to $\mathrm{R}$-CHOP scheme had distinctive differences in $5 \mathrm{hmC}$ enrichment, containing 205 DhMRs detected by differential analysis method. Additionally, pathway analysis of the 205 marker genes with differentially modified $5 \mathrm{hmC}$ between responders and nonresponders suggested enrichment in alpha-beta $\mathrm{T}$ cell activation and differentiation signaling pathway. As we all known, tumor progression and drug resistance are highly associated with the physiological state of the tumor microenvironment (TME), and thus, the tumor microenvironment (TME) represents an attractive therapeutic target and closely related to the curative effect of tumor therapy [47]. The composition of tumor microenvironment is complex, which mainly include tumor cells, stromal elements, extracellular matrixes, inflammation, and immune cells [48], which are closely related to tumor development, metastasis, and tumor therapy [49]. Importantly, cfDNA is not only derived from tumor cells, but also from the tumor microenvironment [51]. Therefore, these $5 \mathrm{hmC}$ marker genes could be related to the effect of $\mathrm{R}-\mathrm{CHOP}$ treatment.

Furthermore, we found that $135 \mathrm{hmC}$ markers filtered by machine learning algorithms could well distinguish non-responders from responders in both the training and validation cohorts. Meantime, the prediction performance of the logistic regression model, established by $135 \mathrm{hmC}$ markers, achieving 0.82 sensitivity and 0.75 specificity (AUC $=0.78$, was superior to existing clinical indicators, such as LDH $(\mathrm{AUC}=0.646)$ and stage $(\mathrm{AUC}=0.658)$. Furthermore, when combining the LDH and stage, the AUC was also lower than $135 \mathrm{hmC}$ markers. Taken together, these findings indicated that $5 \mathrm{hmC}$ markers derived from cfDNA may serve as effective biomarkers for

(See figure on next page.)

Fig. 5 ARHGEF12 and its potential relevance in DLBCL patients and treatment response. a Boxplot of ARHGEF12 grouped by treatment response (PDSD vs PRCR). Log2 transformed of TMM normalized $5 \mathrm{hmC}$ enrichment values were plotted, and Wilcoxon $t$ test was used. b Functional protein-protein interaction networks (PPI) from the STRING database. $\mathbf{c}-\mathbf{h}$ Correlation plots of the mRNA expression of ARHGEF12 with the mRNA expressions of genes in the RHO pathway, including RHOA, RHOB, CDC42, ROCK1, GNA12 and GNA13 in DLBCL in the TCGA-DLBC dataset. i, j Overall survival curves of DLBCL patients with low or high gene expressions in ARHGEF12 or CDC42 in the TCGA-DLBC dataset. The $x$-axis represents the OS time (days), and the $y$-axis represents the survival probability. (K) GO enrichment bar plot for genes associated with ARHGEF12 as shown in the PPI network $\left({ }^{*} p=0.005-0.05,{ }^{* *} p=0.0005-0.005\right)$ 
a

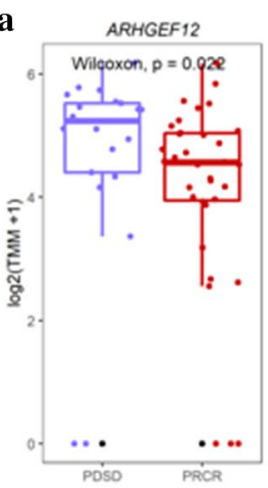

d

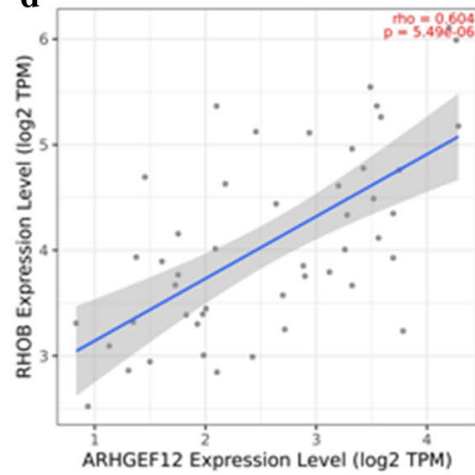

g

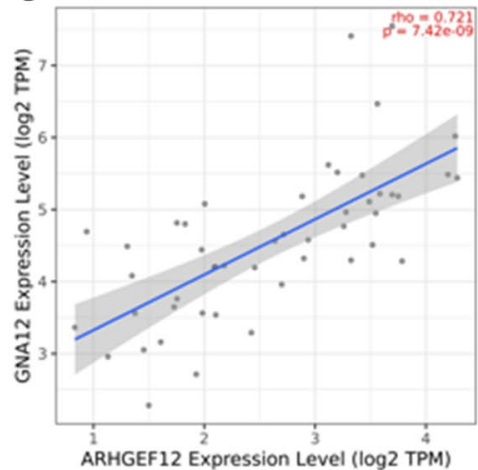

j

j $\operatorname{COC} 42_{2}+$ High - Low

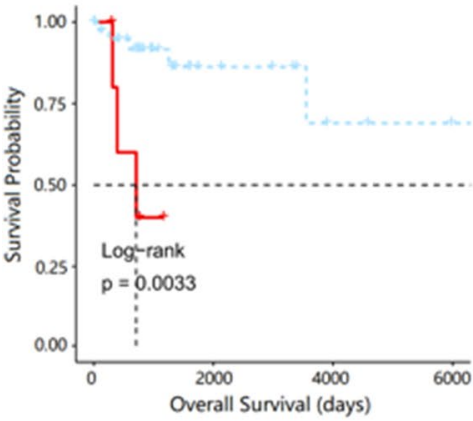

b
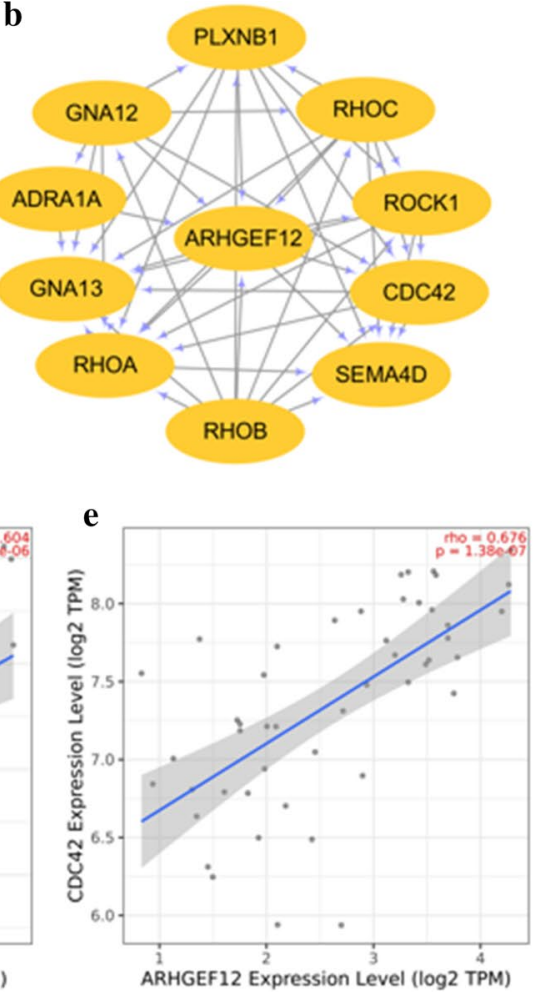

h

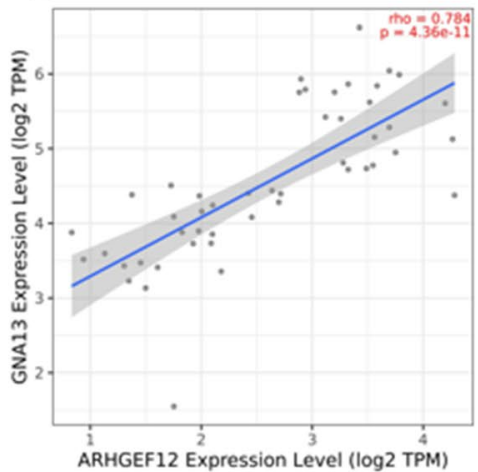

k

Activation of Rho by LARG and PDZ-RhoGEF GDIs block activation of Rho GTPase:GDP

Rho GTPase:GTP activates downstream effectors GEFs activate Rho GTPase:GDP

GAPs inactivate Rho GTPase: GTP by hydrolysis Dissociation of Rho GTP:GDP from GDI complex ROCK activation by RHO

Myosin phosphatase inactivation by ROCK LIM kinase phosphorylation by ROCK GEFs activate RhOA,B,C Myosin regulatory light chain phosphorylation by ROCK
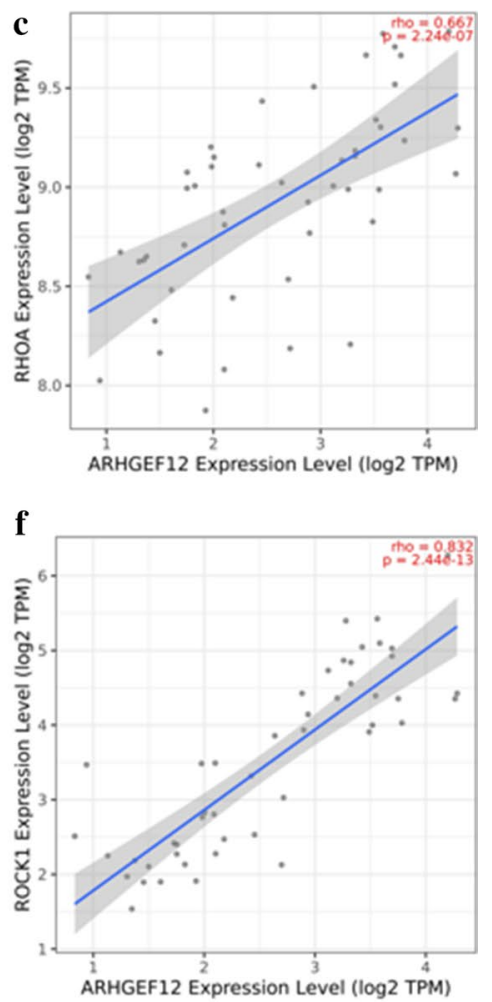

i

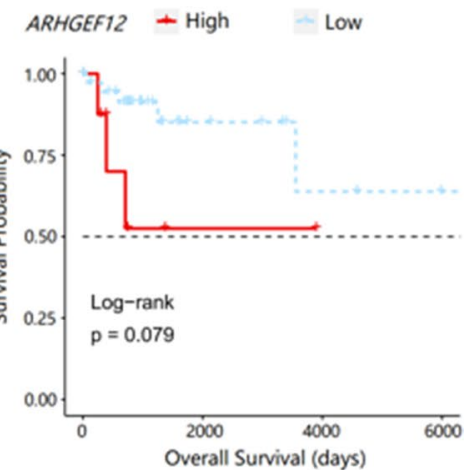

$\%$ Genes / Term

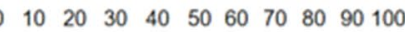

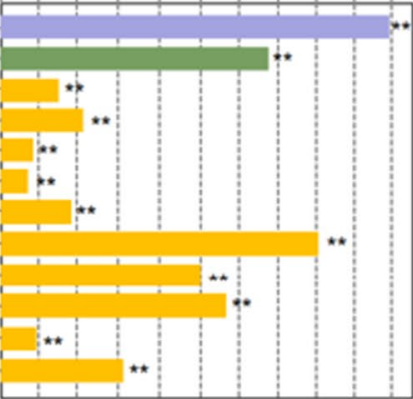


minimally noninvasive prediction for treatment response of DLBCL patients with R-CHOP scheme.

According to recent studies, $5 \mathrm{hmC}$ enrichment in promoter regions can promote gene transcription [25]. In our study, the hydroxymethylation of ARHGEF12 is enriched in the promoter region in non-responders. Notably, among 13 5hmC marker genes, ARHGEF12 showed the best predictive performance, and its mRNA expression was positively associated with that of MS4A1 in the TCGA-DLBC dataset. Meantime, ARHGEF12 expression was highly positively correlated with Rhorelated genes, such as RHOA, RHOB, CDC42, ROCK1, GNA12, and GNA13. Previous research suggested that Rho signaling pathway was linked to cancer microenvironment, cancer initiation, proliferation, and metastasis, and might incorporate novel biological implications and therapeutic opportunities [52]. These genes related to ARHGEF12 all play crucial roles in the Rho signaling pathway, and their functions in cancer initiation, proliferation, metastasis, and drug resistance are well supported by previous research [52-57]. In addition, the potential functions of $A R H G E F 12$ were also reported in various researches. For instance, ARHGEF12 is a wellstudied activator of Rho signaling downstream of G-protein-coupled receptors (GPCRs) and has essential roles in chemokine-driven tumor cell invasion [58, 59]. More importantly, ARHGEF12 expression was also positively associated with immune-related genes, such as $C D 44$, CD47, CD53, CD59, and CD274. Therefore, we suspected that, like its related genes, ARHGEF12 was crucial in the Rho signaling pathway and might be related to diffuse large B cell lymphoma initiation, proliferation, metastasis and treatment, but the deep biological basis of these relationships needs further study. Taken together, the above evidence provided by previous research suggested that ARHGEF12 might serve as a potential drug target that was related to the treatment response of $\mathrm{R}-\mathrm{CHOP}$ treatment.

Nevertheless, this study still has some limitations. First, the sample size is relatively small and may not fully represent all DLBCL patients. The performance of our model still needs to be tested in larger study cohorts. Second, this study only focuses on Chinese patients and may not represent DLBCL patients in other races. Thirdly, the regulatory mechanism of $5 \mathrm{hmC}$ in ARHGEF12 and its relevance in $\mathrm{R}-\mathrm{CHOP}$ treatment effectiveness are still not clear. Thus, further studies are required. In the future, we aim to increase the sample size of DLBCL patients and find more stable and reliable $5 \mathrm{hmC}$ marker genes to predict the treatment response of $\mathrm{R}-\mathrm{CHOP}$ scheme.

In conclusion, our results suggested that $5 \mathrm{hmC}$ markers derived from plasma cfDNA can be used to predict treatment response of DLBCL patients treated with
R-CHOP scheme. Meanwhile, hmC-Seal might serve as a minimally noninvasive technique to unveil potential drug targets related to the treatment response of $\mathrm{R}-\mathrm{CHOP}$ in DLBCL patients.

\section{Supplementary information}

Supplementary information accompanies this paper at https://doi. org/10.1186/s13148-020-00973-8.

Additional file 1. $2055 \mathrm{hmC}$ markers.anotation

Additional file 2. Supplementary material.

Additional file 3.13 5hmC feature markers.anotation.

\section{Abbreviations}

R-CHOP: Rituximab, cyclophosphamide, doxorubicin, vincristine, and prednisone; DLBCL: Diffuse large B cell lymphoma; IPI: International Prognostic Index; AID: Activationinduced cytidine deaminase; 5hmC: 5-Hydroxymethylcytosine; 5mC: 5-Methylcytosine; TET: Ten-eleven translocation; cfDNA: Cell-free DNA; WBC: White blood cell count; LDH: Lactate dehydrogenase; $\beta 2 M G$ : $\beta 2$ Microglobulin; PD: Progressive disease; SD: Stable disease; PR: Partial response; CR: Complete response; hMRs: $5 \mathrm{HmC}$-enriched regions; RFECV: Recursive feature elimination algorithm; ROC: Receiver operating characteristic; AUC: The area under ROC curves; STRING: Search Tool for the Retrieval of Interacting Genes; OS: Overall survival; DSS: Disease-specific survival; DFI: Disease-free interval; PFI: Progression-free interval.

\section{Acknowledgments}

We would like to acknowledge the essential contributions of all staffs and students who participated in this work.

\section{Authors' contributions}

$\mathrm{H}-\mathrm{YC}$ and W-LZ conceived the study and designed the experiments. $\mathrm{H}-\mathrm{YC}$ performed the experiments with the help from LZ. H-YC analyzed data with help from Z-RY. PY, FL, JW, MP, YH, CY, WL, JL, X-BX, YQ, X-HH, and $H L$ recruited patients, collected blood, and organized clinical in formation. H-YC, W-LZ, and Z-RY wrote the manuscript with input and comments from PY, NX, and LC. All authors read and approved the final manuscript.

\section{Funding}

This research was funded by Beijing Natural Science Foundation (7132183 and 7182178), China Health Promotion Foundation (CHPF-zlkysx-001), Key Clinical Projects of Peking University Third Hospital (BYSYZD2019026), Natural Science Foundation of China (81800195, 31471299 and 81522046), and National

Science and Technology Major Projects for "Major New Drugs Innovation and Development" (No. 2018ZX09711003).

\section{Availability of data and materials}

The datasets supporting the conclusions of this article are included within the article and its additional files. All other datasets used and analyzed during the current study are available from the corresponding author on reasonable request.

\section{Ethics approval and consent to participate}

The study was conducted according to the guidelines of the Helsinki Declaration and was approved by the Ethics Committee of Peking University Third Hospital. Written informed consent was obtained from all participants.

Consent for publication

Not applicable.

Competing interests

The authors declare that they have no conflict of interests.

\section{Author details}

${ }^{1}$ Synthetic and Functional Biomolecules Center, Beijing National Laboratory for Molecular Sciences, Key Laboratory of Bioorganic Chemistry and Molecular 
Engineering of Ministry of Education, College of Chemistry and Molecular Engineering, Innovation Center for Genomics, Peking University, Beijing 100871, People's Republic of China. ${ }^{2}$ Department of Hematology, Lymphoma Research Center, Peking University Third Hospital, Beijing 100191, People's Republic of China. ${ }^{3}$ Lymphoma Head and Neck Oncology, Fifth Medical Center of PLA General Hospital, Beijing 100039, People's Republic of China. ${ }^{4}$ Department of Medical Oncology, National Cancer Center/Cancer Hospital, Chinese Academy of Medical Sciences and Peking Union Medical College, Beijing 100021, People's Republic of China. ${ }^{5}$ Department of Hematology, Beijing Hospital, National Center of Gerontology, Beijing 1000730, People's Republic of China. ${ }^{6}$ Yang Sheng Tang Natural Medicine Research Institute, Hangzhou 310024, People's Republic of China. ${ }^{7}$ Department of Chemistry, University of Chicago, Chicago, IL 60637, USA. ${ }^{8}$ Institute of Biology and Medicine, College of Life and Health 20 Sciences, Wuhan University of Science and Technology, Hubei 430081, People's Republic of China.

Received: 22 July 2020 Accepted: 9 November 2020 Published online: 11 February 2021

\section{References}

1. Cheson BD, Fisher Rl, Barrington SF, Cavalli F, Schwartz LH, Zucca E, et al. Recommendations for initial evaluation, staging, and response assessment of Hodgkin and non-Hodgkin lymphoma: the Lugano classifification. J Clin Oncol. 2014;32:3059-67.

2. Oschlies I, et al. Diffuse large B-cell lymphoma in pediatric patients belongs predominantly to the germinal-center type B-cell lymphomas: $A$ clinicopathologic analysis of cases included in the German BFM (BerlinFrankfurt-Munster) Multicenter Trial. Blood. 2006a;107(10):4047-52.

3. Fu K, et al. Addition of rituximab to standard chemotherapy improves the survival of both the germinal center B-cell-like and non-germinal center B-cell-like subtypes of diffuse large B-cell lymphoma. J Clin Oncol. 2008;26(28):4587-94.

4. Coiffier B, Sarkozy C. Diffuse large B-cell lymphoma: R-CHOP failurewhat to do? American Society of Hematology. 2016;1:366-78.

5. Oschlies I, et al. Diffuse large B-cell lymphoma in pediatric patients belongs predominantly to the germinal-center type B-cell lymphomas: a clinicopathologic analysis of cases included in the German BFM (BerlinFrankfurt-Münster) Multicenter Trial. Blood. 2006b;107(10):4047-52.

6. Kim SH, et al. Prognostic impact of pretreatment albumin to globulin ratio in patients with diffuse large B-cell lymphoma treated with R-CHOP. Leuk Res. 2018;71:100-5.

7. Barrington SF, Mikhaeel NG. PET scans for staging and restaging in diffuse large B-cell and follicular lymphomas. Curr Hematologic Malig Rep. 2016;11(3):185-95.

8. International Non-Hodgkin's Lymphoma Prognostic Factors Project. A predictive model for aggressive non-Hodgkin's lymphoma. N Engl J Med. 1993;329(14):987-94.

9. Flowers CR, Sinha R, Vose JM. Improving outcomes for patients with diffuse large B-cell lymphoma. CA Cancer J Clin. 2010;60(6):393-408.

10. Zhou Z, et al. An enhanced International Prognostic Index (NCCN-IPI) for patients with diffuse large B-cell lymphoma treated in the rituximab era. Blood. 2014;123(6):837-42.

11. Deng $\mathrm{Y}$, et al. EZH2/BCl-2 coexpression predicts worse survival in diffuse large B-cell lymphomas and demonstrates poor efficacy to rituximab in localized lesions. J Cancer. 2019;10(9):2006-17.

12. Markovic $\mathrm{O}$, et al. Survivin expression in patients with newly diagnosed nodal diffuse large B cell lymphoma (DLBCL). Med Oncol. 2012;29(5):3515-21.

13. Arima $\mathrm{H}$, et al. Prognostic impact of activation-induced cytidine deaminase expression for patients with diffuse large B-cell lymphoma. Leuk Lymphoma. 2018;59(9):2085-95.

14. Song $G$, et al. Serum microRNA expression profiling predict response to R-CHOP treatment in diffuse large B cell lymphoma patients. Ann Hematol. 2014;93(10):1735-43.

15. Feng $Y$, et al. Exosome-derived miRNAs as predictive biomarkers for diffuse large B-cell lymphoma chemotherapy resistance. Epigenomics. 2019;11(1):35-51.

16. Jin X, et al. Homozygous A polymorphism of the complement C1qA276correlates with prolonged overall survival in patients with diffuse large B cell lymphoma treated with R-CHOP. J Hematol Oncol. 2012;5(1):51.

17. Kim DH, et al. FCGR3A gene polymorphisms may correlate with response to frontline R-CHOP therapy for diffuse large B-cell lymphoma. Blood. 2006;108(8):2720-5.

18. Coutinho R, et al. Revisiting the immune microenvironment of diffuse large B-cell lymphoma using a tissue microarray and immunohistochemistry: robust semi-automated analysis reveals CD3 and FoxP3 as potential predictors of response to R-CHOP. Haematologica. 2015;100(3):363-9.

19. Schwarzenbach H, Hoon DS, Pantel K. Cell-free nucleic acids as biomarkers in cancer patients. Nat Rev Cancer. 2011;11:426-37.

20. Dahl C, Gronbaek K, Guldberg P. Advances in DNA methylation:5-hydroxymethylcytosine revisited. Clin Chim Acta. 2011;412(11-12):831-6.

21. Kristensen $L S$, et al. Aberrant methylation of cell-free circulating DNA in plasma predicts poor outcome in diffuse large B cell lymphoma. Clin Epigenetics. 2016;8(1):95-95.

22. Tahiliani M, Koh KP, Shen Y, Pastor WA, Bandukwala H, Brudno Y, et al. Conversion of 5-methylcytosine to 5-hydroxymethylcytosine in mammalian DNA by MLL partner TET1. Science. 2009;324:930-5.

23. Kriaucionis $\mathrm{S}$, Heintz $\mathrm{N}$. The nuclear DNA base 5 -hydroxymethylcytosine is present in Purkinje neurons and the brain. Science. 2009;324:929-30.

24. FuY, He C. Nucleic acid modififications with epigenetic signifificance. Curr Opin Chem Biol. 2012;16:516-24.

25. Branco MR, Ficz G, ReikW. Uncovering the role of 5-hydroxymethylcytosine in the epigenome. Nat Rev Genet. 2011;13:7-13.

26. Chiu BCH, et al. Prognostic implications of 5-hydroxymethylcytosines from circulating cell-free DNA in diffuse large B-cell lymphoma. Blood Adv. 2019;3(19):2790-9.

27. Fang $C, X u W$, et al. A systematic review and meta-analysis of rituximabbased immunochemotherapy for subtypes of diffuse large B cell lymphoma. Ann Hematol. 2010;89:1107-13.

28. Van Heertum RL, Scarimbolo R, et al. Lugano 2014 criteria for assessing FDG-PET/ CT in lymphoma: an operational approach for clinical trials. Drug Des Dev Therapy. 2014;2017(11):1719-28.

29. Song CX, Szulwach KE, Fu Y, et al. Selective chemical labeling reveals the genome-wide distribution of 5-hydroxymethylcytosine. Nat Biotechnol. 2011;29:68-72.

30. Langmead B, Salzberg SL. Fast gapped-read alignment with Bowtie 2. Nat Methods. 2012;9:357-9.

31. Zhang Y, et al. Model-based analysis of ChIP-Seq (MACS). Genome Biol. 2008;9(9):137.

32. Quinlan AR, Hall IM. BEDTools: a flexible suite of utilities for comparing genomic features. Bioinformatics. 2010;26:841-2.

33. Zhang Y, Liu T, Meyer CA, et al. Model-based analysis of ChIP-Seq (MACS). Genome Biol. 2008;9:R137.

34. Pedregosa F, Varoquaux G, Gramfort A, Michel V, Thirion B, Grisel O, et al. Scikit-learn: machine learning in Python. J Mach Learn Res. 2011;12:2825-30

35. McCarthy DJ, Chen Y, Smyth GK. Differential expression analysis of multifactor RNA-Seq experiments with respect to biological variation. Nucleic Acids Res. 2012;40(10):4288-97.

36. Yu G, Wang LG, He QY. ChIPseeker: an R/Bioconductor package for ChIP peak annotation, comparison and visualization. Bioinformatics. 2015;31(14):2382-3.

37. Schmitz R, Wright GW, Huang DW, Johnson CA, Phelan JD, Wang JQ, Roulland S, Kasbekar M, Young RM, Shaffer AL, Hodson DJ. Genetics and pathogenesis of diffuse large B-cell lymphoma. N Engl J Med. 2018;378(15):1396-407.

38. Goldman M, Craft B, Brooks A, Zhu J, Haussler D. The UCSC Xena Platform for cancer genomics data visualization and interpretation. BioRxiv 2018; 326470.

39. Hothorn T, Hothorn MT, Suggests TH. Package 'maxstat'. 2017.

40. Goel MK, Khanna P, Kishore J. Understanding survival analysis: KaplanMeier estimate. Int J Ayurveda Res. 2010;1 (4):274.

41. Marubini E, Valsecchi MG. Estimation of survival probabilities. Analysing survival data from clinical trials and observational studies. Chichester: Wiley; 1995. p. 41-8. 
42. Li T, Fan J, Wang B, Traugh N, Chen Q, Liu JS, Li B, Liu XS. TIMER: a web server for comprehensive analysis of tumor-infiltrating immune cells. Cancer Res. 2017;77(21):e108-10.

43. McKight PE, Najab J. Kruskal-Wallis test. The Corsini Encyclopedia of Psychology 2010;1-1.

44. Cochran WG. The $\times 2$ test of goodness of fit. Ann Math Stat. 1952;23:315-45.

45. Applebaum MA, Barr EK, et al. 5-Hydroxymethylcytosine profifiles are prognostic of outcome in neuroblastoma and reveal transcriptional networks that correlate with tumor phenotype. JCO Precis Oncol. 2019;3:1-12.

46. Zhang Ji, Han X, et al. 5-hydroxymethylome in circulating cell-free DNA as A potential biomarker for non-small-cell lung cancer. Genomics Proteomics Bioinformatics. 2018;16:187-99.

47. Quail DF, Joyce JA. Microenvironmental regulation of tumor progression and metastasis. Nat Med. 2013;19(11):1423-37.

48. Alexandra IC, Patricia IS, Liana S, et al. Tumor microenvironment in diffuse large B-cell lymphoma: role and prognosis. Anal Cell Pathol. 2019;2019:8586354.

49. Catarina RR, Rita M. Targeting tumor microenvironment for cancer therapy. Int J Mol Sci. 2019;20:840.

50. Song C-X, et al. 5-Hydroxymethylcytosine signatures in cell-free DNA provide information about tumor types and stages. Cell Res. 2017:27(10):1231-42.

51. Swaminathan R, Butt AN. Circulating nucleic acids in plasma and serum: recent developments. Ann NY Acad Sci. 2006;1075:1-9.

52. Venessa T, Adnan M, et al. Rho-associated kinase signalling and the cancer microenvironment: novel biological implications and therapeutic opportunities. Expert Rev Mol Med. 2015;17:1-14.
53. Müller PM, Rademacher J, Bagshaw RD, et al. Systems analysis of RhoGEF and RhoGAP regulatory proteins reveals spatially organized RAC 1 signalling from integrin adhesions. Nat Cell Biol. 2020;22(4):498-511.

54. Patrick K, Stemmle LN, Madden JF, et al. A role for the G12 family of heterotrimeric $\mathrm{G}$ proteins in prostate cancer invasion. J Biol Chem. 2006;281(36):26483-90.

55. Kelly $\mathrm{P}$, et al. The $\mathrm{G} 12$ family of heterotrimeric $\mathrm{G}$ proteins promotes breast cancer invasion and metastasis. PNAS. 2006;103(21):8173-8.

56. Yuan Bo, et al. Ga12/13 signaling promotes cervical cancer invasion through the RhoA/ROCK-JNK signaling axis. Biochem Biophys Res Commun. 2016;473(4):1240-6.

57. Suhail $A$, Hui Sun $L$, et al. GNA13 expression promotes drug resistance and tumor-initiating phenotypes in squamous cell cancers. Oncogene. 2018:37:1340-53.

58. Yagi $H$, et al. A synthetic biology approach reveals a CXCR4-G13-Rho signaling axis driving transendothelial migration of metastatic breast cancer cells. Sci Signal. 2011;4:ra60.

59. Struckhof AP, et al. PDZ-RhoGEF is essential for CXCR4-driven breast tumor cell motility through spatial regulation of RhoA. J Cell Sci. 2013;126:4514-26.

\section{Publisher's Note}

Springer Nature remains neutral with regard to jurisdictional claims in published maps and institutional affiliations.
Ready to submit your research? Choose BMC and benefit from:

- fast, convenient online submission

- thorough peer review by experienced researchers in your field

- rapid publication on acceptance

- support for research data, including large and complex data types

- gold Open Access which fosters wider collaboration and increased citations

- maximum visibility for your research: over $100 \mathrm{M}$ website views per year

At $\mathrm{BMC}$, research is always in progress.

Learn more biomedcentral.com/submissions 